\title{
Gas-Phase Synthesis, Characterization, and Reactivity Studies of the Prototype Aromatic Phosphirenylium Ion
}

\author{
Xinping Liu, Diana Ivanova, Daryl Giblin, Michael L. Gross*, Peter P. Gaspar*
}

\section{Contribution from}

\section{Department of Chemistry, Washington University}

St. Louis, MO 63130-4899

\section{Submitted to}

\section{Organometallics}

*Corresponding authors. E-mails: gaspar@wuchem.wustl.edu, mgross@wustl.edu

Supporting Materials. (1) A complete listing of Cartesian coordinates for each optimized structure and computer generated structures at the B3LYP/6-31G* level of theory are available as supplementary material; (2) Table S1: Total and relative energies of $\mathrm{C}_{2} \mathrm{H}_{2} \mathrm{P}^{+}$isomers at the G3//B3LYP/6-31G* level; (3) Table S2: Total energies of species involved in the potential energy surfaces of the reaction of $\mathrm{PBr}^{+}$with $\mathrm{C}_{2} \mathrm{H}_{2}$ at the B3LYP/6-311+G**//B3LYP/6-31G* and G3//B3LYP/6-31G* levels; (4) Table S3: Total energies of species involved in the potential energy surfaces of the reaction of $\mathrm{C}_{2} \mathrm{H}_{2} \mathrm{P}^{+}$with $\mathrm{C}_{4} \mathrm{H}_{6}$ at the G3//B3LYP/6-31G* level. 
1. 1-Singlet $\mathrm{C}_{2} \mathrm{H}_{2} \mathrm{P}^{+}$

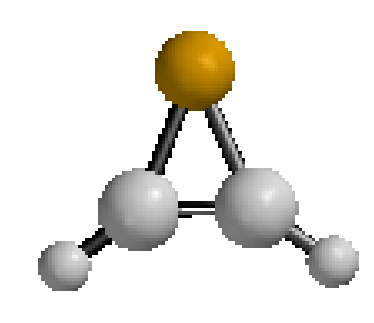

Atom

Cartesian Coordinates (Angstroms)

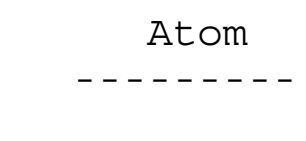

$\begin{array}{lll}1 & \mathrm{C} & \mathrm{C} 1\end{array}$

$2 \mathrm{H} \quad \mathrm{H} 2$

$3 \mathrm{C} \quad \mathrm{C} 2$

$4 \mathrm{H} \quad \mathrm{H} 4$

5 P P1

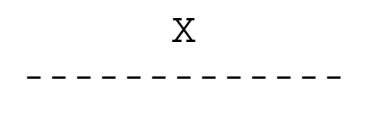

0.6747372

0.0641068

0.0000000

1.5516157

0.7048361

0.0000000

$-0.6748136$

0.0639724

0.0000000

$-1.5518401$

0.7044916

0.0000000

0.0003007

$-1.5374069$

0.0000000

\section{2-Singlet $\mathrm{C}_{2} \mathrm{H}_{2} \mathrm{P}^{+}$}

\begin{tabular}{lll} 
& & \multicolumn{1}{c}{ Atom } \\
& & \\
1 & $\mathrm{C}$ & $\mathrm{C} 1$ \\
2 & $\mathrm{H}$ & $\mathrm{H} 2$ \\
3 & $\mathrm{H}$ & $\mathrm{H} 3$ \\
4 & $\mathrm{C}$ & $\mathrm{C} 2$ \\
5 & $\mathrm{P}$ & $\mathrm{P} 1$
\end{tabular}

\begin{tabular}{ccc} 
Cartesian Coordinates & \multicolumn{2}{c}{ (Angstroms) } \\
X & Y & Z \\
& & \\
0.0000000 & 0.0000000 & 0.6275760 \\
0.9358014 & 0.0000000 & 1.1927314 \\
-0.9358014 & 0.0000000 & 1.1927314 \\
0.0000000 & 0.0000000 & -0.7145246 \\
0.0000000 & 0.0000000 & -2.2985142
\end{tabular}




\section{3-Singlet $\mathrm{C}_{2} \mathrm{H}_{2} \mathrm{P}^{+}$}

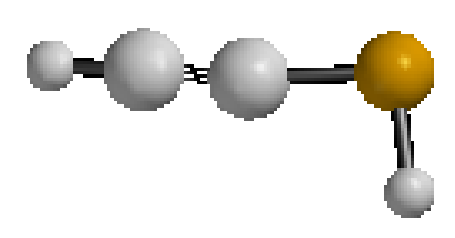

Atom

Cartesian Coordinates (Angstroms) - - - - - - -

$\begin{array}{lll}1 & \mathrm{C} & \mathrm{C} 1\end{array}$

$2 \mathrm{C} \quad \mathrm{C} 2$

$3 \mathrm{H} \quad \mathrm{H} 2$

4 P $\mathrm{P} 1$

$5 \mathrm{H} \quad \mathrm{H} 3$
$\mathrm{X}$

- - - - - - - - - - -

$-0.0551360$

$-1.2745063$

$-2.3344300$

1. 5744383

2.0896340
$\mathrm{Y}$

$$
\begin{array}{r}
-0.1974801 \\
-0.0110388 \\
0.1801792 \\
-0.6530313 \\
0.6813710
\end{array}
$$

Z
0.0000000
0.0000000
0.0000000
0.0000000
0.0000000

\section{4-Triplet $\mathrm{C}_{2} \mathrm{H}_{2} \mathrm{P}^{+}$}

\section{Atom}

$\begin{array}{lll}1 & \mathrm{H} & \mathrm{H} 0 \\ 2 & \mathrm{C} & \mathrm{C} 1 \\ 3 & \mathrm{H} & \mathrm{H} 2 \\ 4 & \mathrm{C} & \mathrm{C} 2 \\ 5 & \mathrm{P} & \mathrm{P} 1\end{array}$

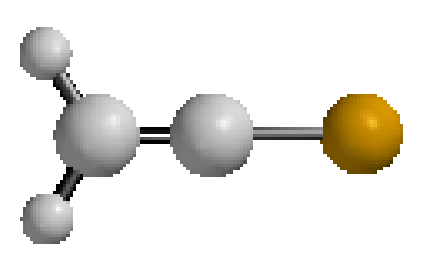

\section{Cartesian Coordinates (Angstroms) $\mathrm{X}$ \\ $\mathrm{Z}$}

$\begin{array}{rrr}-0.9352441 & 0.0000000 & 1.2051355 \\ 0.0000000 & 0.0000000 & 0.6319071 \\ 0.9352441 & 0.0000000 & 1.2051355 \\ 0.0000000 & 0.0000000 & -0.6742293 \\ 0.0000000 & 0.0000000 & -2.3679489\end{array}$




\title{
5. 5-Triplet $\mathrm{C}_{2} \mathrm{H}_{2} \mathrm{P}^{+}$
}

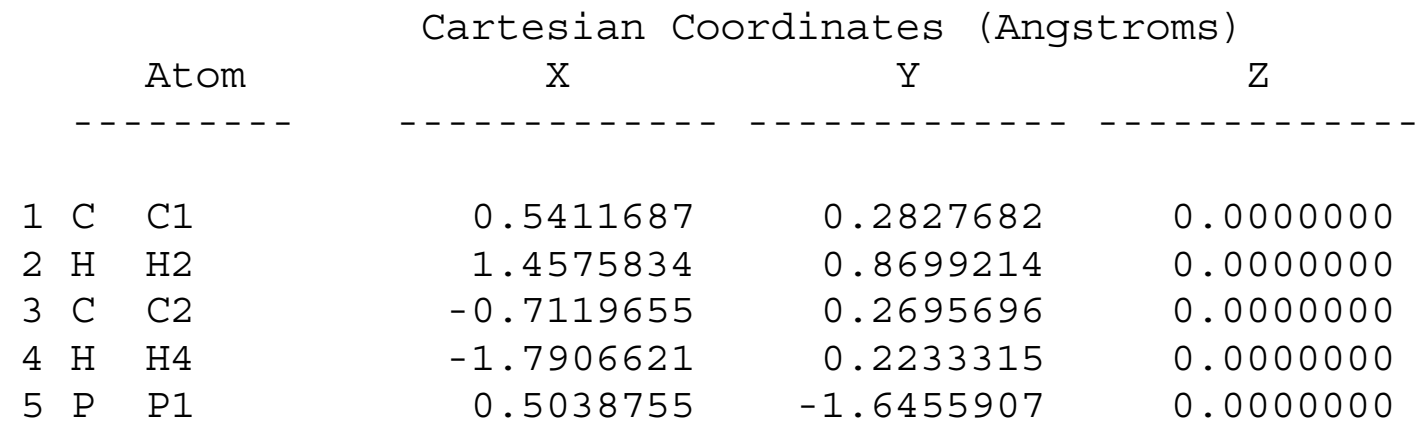

6. 6-Triplet $\mathrm{C}_{2} \mathrm{H}_{2} \mathrm{P}^{+}$

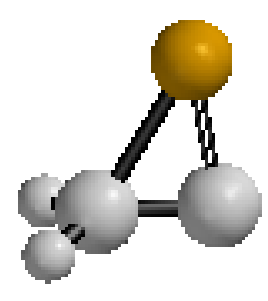

Atom

Cartesian Coordinates (Angstroms)

- Atom

\begin{tabular}{lll}
1 & $\mathrm{C}$ & $\mathrm{C} 1$ \\
\hline
\end{tabular}

$2 \mathrm{H} H 3$

$3 \mathrm{H} H 4$

$\begin{array}{lll}4 & \mathrm{C} & \mathrm{C} 2\end{array}$

5 P P1

\begin{abstract}
0.3912384
0.9483306

0.9483306

$-0.6847758$

$-1.6031238$
\end{abstract}

0.0453190

0.0000000

$-0.1821784$

$-0.9227582$

$-0.1821784$

0.9227582

0.9138771

0.0000000

$-0.5948393$

0.0000000 


\section{7-Triplet $\mathrm{C}_{2} \mathrm{H}_{2} \mathrm{P}^{+}$}

$\begin{array}{lll} & & \text { Atom } \\ 1 & \mathrm{C} & \mathrm{C} 1 \\ 2 & \mathrm{H} & \mathrm{H} 2 \\ 3 & \mathrm{C} & \mathrm{C} 2 \\ 4 & \mathrm{P} & \mathrm{P} 1 \\ 5 & \mathrm{H} & \mathrm{H} 3\end{array}$

\section{8-Singlet $\mathrm{C}_{2} \mathrm{H}_{2} \mathrm{P}^{+}$}

$\begin{array}{lll} & & \text { Atom } \\ 1 & \mathrm{P} & \mathrm{P} 1 \\ 2 & \mathrm{H} & \mathrm{H} 2 \\ 3 & \mathrm{H} & \mathrm{H} 3 \\ 4 & \mathrm{C} & \mathrm{C} 1 \\ 5 & \mathrm{C} & \mathrm{C} 2\end{array}$

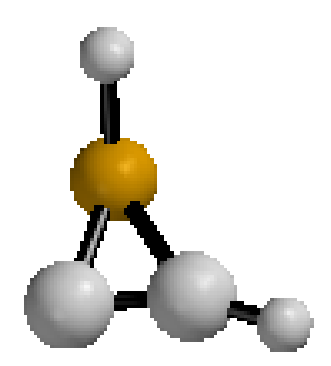

Cartesian Coordinates (Angstroms)

\begin{tabular}{|c|c|c|}
\hline $\mathrm{X}$ & $\mathrm{Y}$ & Z \\
\hline------------ & ------------ & ------------- \\
\hline 0.8377980 & 0.0157087 & -0.0186691 \\
\hline 1.6583957 & 0.7254784 & 0.0285947 \\
\hline 0.2872071 & -1.1572513 & 0.2160793 \\
\hline-0.9173106 & -0.1133107 & -0.5434194 \\
\hline-1.8660902 & 0.5293748 & 0.3174146 \\
\hline
\end{tabular}




\section{9-Triplet $\mathrm{C}_{2} \mathrm{H}_{2} \mathrm{P}^{+}$}

\begin{tabular}{lllrrr} 
& & Cartesian & Coordinates & \multicolumn{2}{c}{ (Angstroms) } \\
& & Atom & X & Z & \multicolumn{1}{c}{ Z } \\
& & & & & \\
1 & H & H0 & -1.2791760 & -0.1427578 & 1.1624065 \\
2 & P & P1 & -0.6603335 & 0.3496412 & 0.0000000 \\
3 & H & H3 & -1.2791760 & -0.1427578 & -1.1624065 \\
4 & C & C1 & 0.9919879 & -0.0068385 & 0.0000000 \\
5 & C & C2 & 2.2266977 & -0.0572871 & 0.0000000
\end{tabular}

10. 10-Singlet $\quad \mathrm{C}_{2} \mathrm{H}_{2} \mathrm{P}^{+}$

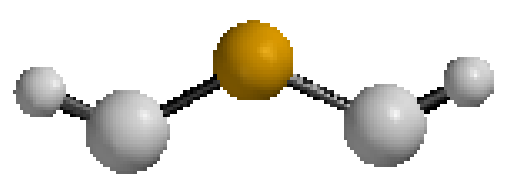

Atom

Cartesian Coordinates (Angstroms)

- - - - - - -

$1 \mathrm{C} \quad \mathrm{C} 1$

$2 \mathrm{H} \quad \mathrm{H} 2$

3 P $\mathrm{P} 1$

$4 \mathrm{C} \quad \mathrm{C} 2$

$5 \mathrm{H} \quad \mathrm{HI}$

1.4811989
2.4642223
0.0000000
-1.4811989
-2.4642223

0.0000000

0.3471872

0.0000000

$-0.1355928$

0.0000000

$-0.4231888$

0.0000000

0.3471872

0.0000000

$-0.1355928$ 


\section{11-Singlet $\mathrm{C}_{2} \mathrm{H}_{2}$}

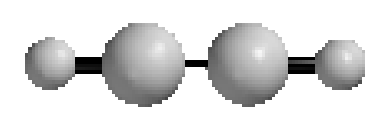

Atom

Cartesian Coordinates (Angstroms)

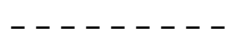

$$
\mathrm{X}
$$

$Y$

$\mathrm{Z}$

$\begin{array}{lll}1 & \mathrm{H} & \mathrm{H} 1 \\ 2 & \mathrm{C} & \mathrm{C} 1 \\ 3 & \mathrm{C} & \mathrm{C} 2 \\ 4 & \mathrm{H} & \mathrm{H} 2\end{array}$

12. 12-Doublet $\mathrm{BrP}^{+}$

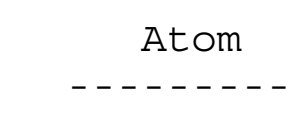

$\begin{array}{lll}1 & \mathrm{P} & \mathrm{P} 1\end{array}$

2 Br Brl

\begin{abstract}
0.0000000
0.0000000

0.0000000

0.0000000
\end{abstract}

0.0000000

0.0000000

0.0000000

0.0000000
$-1.6692066$
$-0.6025366$
0.6025366
1.6692066

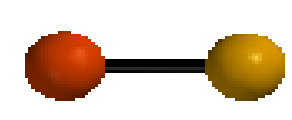

$\begin{array}{ccc}\text { Cartesian Coordinates } & \text { (Angstroms) } \\ \mathrm{X} & \mathrm{Y} & \mathrm{Z} \\ & & \\ 0.0000000 & 0.0000000 & 1.0443713 \\ 0.0000000 & 0.0000000 & -1.0443713\end{array}$

Cartesian Coordinates (Angstroms)

0.0000000

0.0000000

$-1.0443713$

\section{13-Doublet $\mathrm{Br}$}

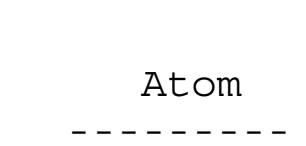

1 Br Brl
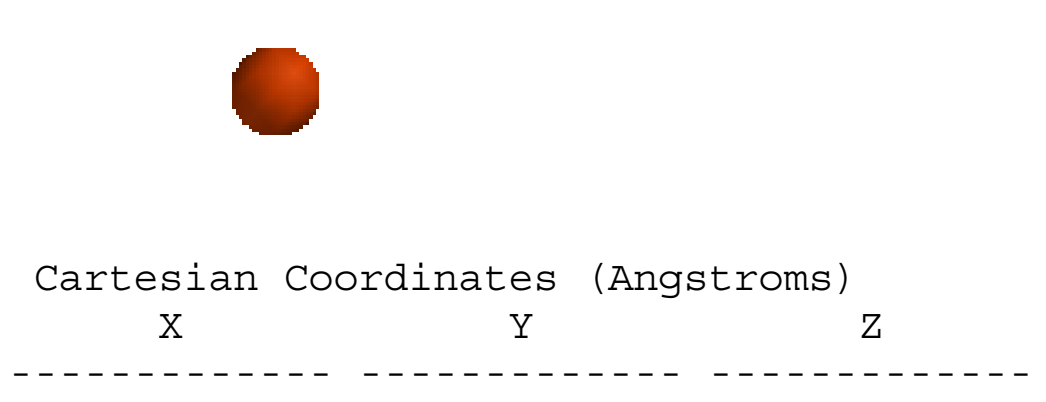
0.0000000
0.0000000
0.0000000 
14. 14-Doublet $\mathrm{C}_{2} \mathrm{H}_{2} \mathrm{BrP}^{+}$

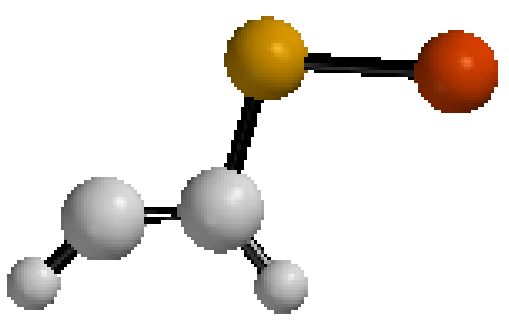

Atom

Cartesian Coordinates (Angstroms)

- - - - - -

$\begin{array}{lll}1 & \mathrm{C} & \mathrm{C} 1 \\ 2 & \mathrm{H} & \mathrm{H} 2 \\ 3 & \mathrm{C} & \mathrm{C} 2 \\ 4 & \mathrm{H} & \mathrm{H} 4 \\ 5 & \mathrm{P} & \mathrm{P} 1 \\ 6 & \mathrm{Br} & \mathrm{Br} 1\end{array}$

0.1760938
-0.0047246
1.3958239
2.4535301
-1.1019185
-2.9188046

0.1743463

0.0000000

1.2556238

0.0000000

$-0.3735445$

0.0000000

$-0.1270370$

0.0000000

$-1.0481567$

0.0000000

$-2.9188046$

0.1187681

0.0000000

15. 15-Doublet T.S. $\mathrm{C}_{2} \mathrm{H}_{2} \mathrm{BrP}^{+}$

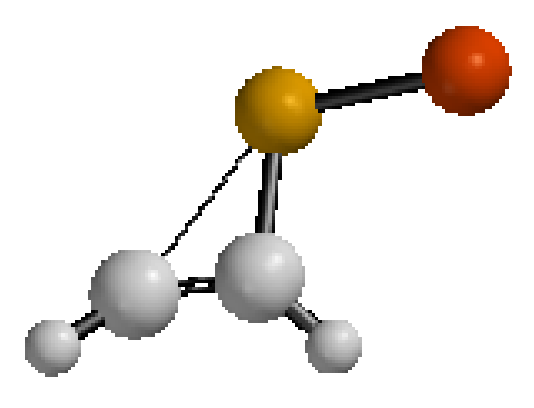

Atom

$\begin{array}{ccr}\text { Cartesian Coordinates } & \text { (Angstroms) } \\ \mathrm{X} & \mathrm{Y} & \mathrm{Z}\end{array}$

$\begin{array}{lll}1 & \mathrm{C} & \mathrm{C} 1 \\ 2 & \mathrm{H} & \mathrm{H} 2 \\ 3 & \mathrm{C} & \mathrm{C} 2 \\ 4 & \mathrm{H} & \mathrm{H} 4 \\ 5 & \mathrm{P} & \mathrm{P} 1 \\ 6 & \mathrm{Br} & \mathrm{Br} 1\end{array}$

0.1835943
-0.0090566
1.3338522
2.3713642
-1.0196536
-2.8601006

0.3144060

$-0.1899056$

1.3894000

$-0.1041502$

$-0.3137141$

$-0.3401828$

$-0.0175440$

0.2947491

$-0.9904332$

$-0.3224431$

$-0.0594759$

0.3392938 
16. 16-Doublet $\mathrm{C}_{2} \mathrm{H}_{2} \mathrm{BrP}^{+}$

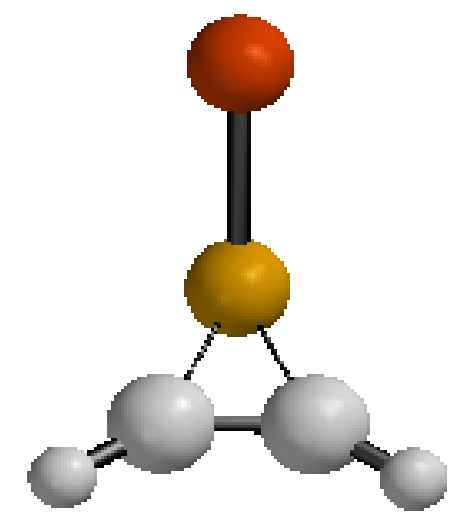

Atom

Cartesian Coordinates (Angstroms)

$X \quad Y \quad Z$

$\begin{array}{rllrrr}1 & \mathrm{H} & \mathrm{H} 1 & 1.2188103 & -0.2158780 & -1.5372614 \\ 2 & \mathrm{C} & \mathrm{C} 1 & 0.6217087 & 0.0590764 & -0.6730161 \\ 3 & \mathrm{C} & \mathrm{C} 2 & 0.6217087 & 0.0590764 & 0.6730161 \\ 4 & \mathrm{H} & \mathrm{H} 2 & 1.2188103 & -0.2158780 & 1.5372614 \\ 5 & \mathrm{P} & \mathrm{P} 1 & -0.8704480 & 0.6876723 & 0.0000000 \\ 6 & \mathrm{Br} & \mathrm{Br} 1 & -2.8105900 & -0.3740692 & 0.0000000\end{array}$

17. 17-Doublet T.S. $\quad \mathrm{C}_{2} \mathrm{H}_{2} \mathrm{BrP}^{+}$

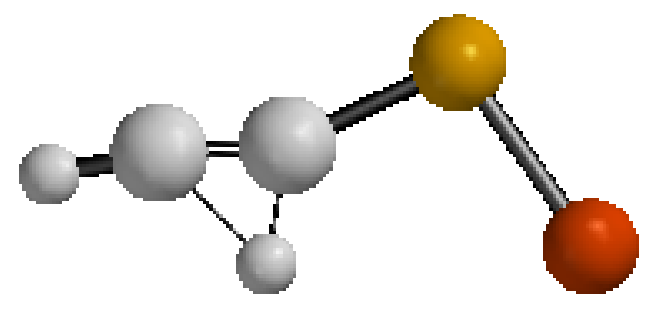

Atom

Cartesian Coordinates (Angstroms)

$\mathrm{X}$

$\mathrm{Y}$

$\mathrm{Z}$

$\begin{array}{lllrrr}1 & \text { P } & \text { P1 } & 1.4885713 & -1.1156937 & -0.0125120 \\ 2 & \text { Br } & \text { Br1 } & 2.8200940 & 0.6358898 & 0.0215204 \\ 3 & \text { C } & \text { C1 } & -0.1458894 & -0.2814506 & -0.0026525 \\ 4 & \text { C } & \text { C2 } & -1.3928143 & -0.1761792 & 0.0085415 \\ 5 & \text { H } & \text { H5 } & -2.4483819 & 0.0552309 & 0.0166190 \\ 6 & \text { H } & \text { H1 } & -0.3215798 & 0.8822030 & -0.0315163\end{array}$


18. 18-Doublet $\mathrm{C}_{2} \mathrm{H}_{2} \mathrm{BrP}^{+}$

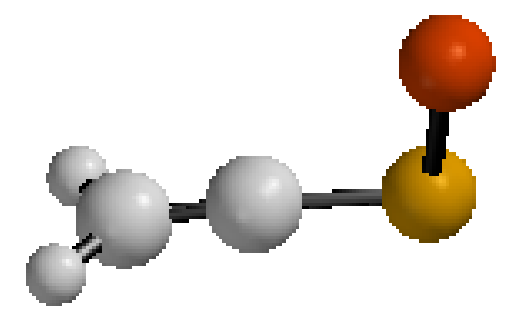

Atom

Cartesian Coordinates (Angstroms) $\mathrm{X}$

$Y$

$\mathrm{Z}$

$\begin{array}{lll}1 & \mathrm{C} & \mathrm{C} 1\end{array}$

$2 \mathrm{H} \quad \mathrm{H} 2$

$3 \mathrm{H} H 3$

$\begin{array}{lll}4 & \mathrm{C} & \mathrm{C} 2\end{array}$

$\begin{array}{lll}5 & \mathrm{P} & \mathrm{P} 1\end{array}$

6 Br Brl

1.1682712
1.8310878
1.6014787
-0.0799130
-1.6411036
-2.8798211

$-0.0506363$

$-0.0134090$

0.5573956

$-0.6387161$

$-0.9461478$

0.4451024

0.2649306

0.1667005

0.8697151

0.4858498

$-0.6952571$

$-0.4455276$

\section{19-Singlet $\mathrm{C}_{4} \mathrm{H}_{4} \mathrm{P}^{+}$}

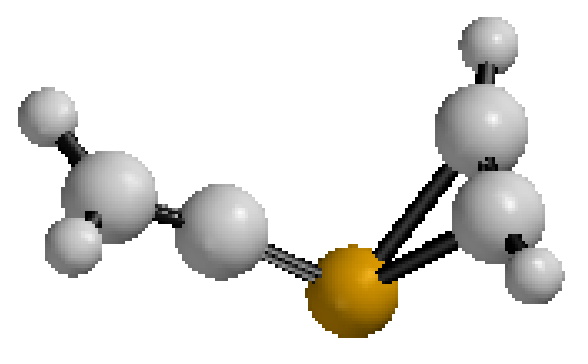

Atom

Cartesian Coordinates (Angstroms) $\mathrm{X}$ $Y$

$1 \mathrm{C} \quad \mathrm{C} 1$

$2 \mathrm{H} \quad \mathrm{H} 2$

$3 \mathrm{C} \quad \mathrm{C} 2$

$4 \mathrm{H} \quad \mathrm{H} 4$

$\begin{array}{lll}5 & \mathrm{P} & \mathrm{P} 1\end{array}$

$6 \mathrm{C} \quad \mathrm{C} 3$

$\begin{array}{lll}7 & \mathrm{C} & \mathrm{C} 4\end{array}$

$8 \mathrm{H} H 1$

$9 \mathrm{H} \mathrm{H5}$
1. 7904523

2.0028084

1. 7904523

2. 0028084

0.5208260

$-0.8845812$

$-2.0646741$

$-2.5790461$

$-2.5790461$

$$
\begin{array}{r}
0.1926740 \\
0.3635466 \\
0.1926740 \\
0.3635466 \\
-1.3784143 \\
-0.4629243 \\
0.0818565 \\
0.3235205 \\
0.3235205
\end{array}
$$




\section{20-Singlet $\mathrm{C}_{4} \mathrm{H}_{4} \mathrm{P}^{+}$}

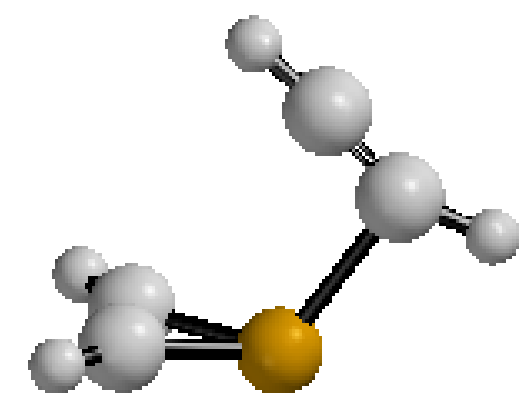

Atom

Cartesian Coordinates (Angstroms) $\mathrm{X}$

$\mathrm{Y}$

$\mathrm{Z}$

$\begin{array}{rrrrrr}1 & \mathrm{C} & \mathrm{C} 1 & 1.8013580 & 0.3823425 & 0.0000000 \\ 2 & \mathrm{H} & \mathrm{H} 2 & 2.4297840 & 1.2578224 & 0.0000000 \\ 3 & \mathrm{C} & \mathrm{C} 2 & 1.6412757 & -0.8346819 & 0.0000000 \\ 4 & \mathrm{H} & \mathrm{H} 4 & 1.3550167 & -1.8693179 & 0.0000000 \\ 5 & \mathrm{P} & \mathrm{P} 1 & -0.2287722 & 1.2028035 & 0.0000000 \\ 6 & \mathrm{H} & \mathrm{H} 1 & -1.9960935 & -0.2274429 & 1.5471741 \\ 7 & \mathrm{C} & \mathrm{C} 3 & -1.5032377 & 0.1579587 & 0.6614615 \\ 8 & \mathrm{C} & \mathrm{C} 4 & -1.5032377 & 0.1579587 & -0.6614615 \\ 9 & \mathrm{H} & \mathrm{H} 3 & & -0.2274429 & -1.5471741\end{array}$

21. 21-Singlet T.S. $\mathrm{C}_{4} \mathrm{H}_{4} \mathrm{P}^{+}$

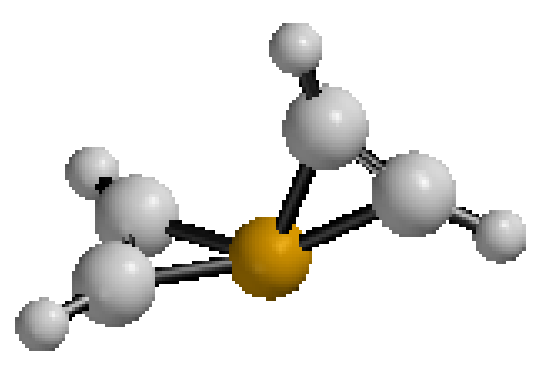


Atom

Cartesian Coordinates (Angstroms) $\mathrm{X}$ $\mathrm{Y}$

Z

$\begin{array}{lll}1 & \mathrm{C} & \mathrm{C} 1 \\ 2 & \mathrm{H} & \mathrm{H} 2 \\ 3 & \mathrm{C} & \mathrm{C} 2 \\ 4 & \mathrm{H} & \mathrm{H} 4 \\ 5 & \mathrm{P} & \mathrm{P} 1 \\ 6 & \mathrm{H} & \mathrm{H} 1 \\ 7 & \mathrm{C} & \mathrm{C} 3 \\ 8 & \mathrm{C} & \mathrm{C} 4 \\ 9 & \mathrm{H} & \mathrm{H} 3\end{array}$

22. 22-Singlet $\mathrm{C}_{4} \mathrm{H}_{4} \mathrm{P}^{+}$

$\begin{array}{rrr}1.3313502 & 0.7288008 & -0.0138599 \\ 1.4329176 & 1.8085301 & -0.0364725 \\ 1.9431345 & -0.3846784 & 0.0085377 \\ 2.6794783 & -1.1781021 & 0.0235878 \\ 0.0165184 & -0.7122641 & 0.0176716 \\ -2.1686077 & 0.0717245 & 1.5231279 \\ -1.5379301 & -0.1549592 & 0.6703571 \\ -1.5345829 & -0.1844060 & -0.6645076 \\ -2.1622783 & 0.0053544 & -1.5284421\end{array}$

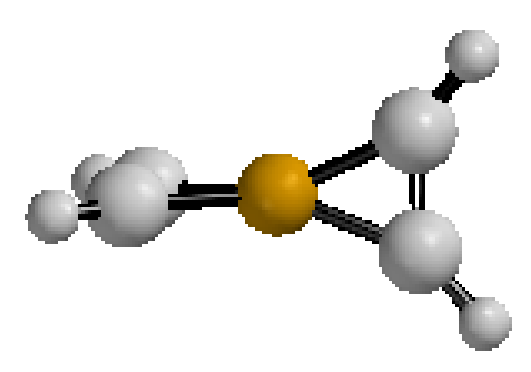

Cartesian Coordinates (Angstroms)

Atom $\mathrm{X}$ $\mathrm{Y}$ Z

$\begin{array}{lll}1 & \mathrm{C} & \mathrm{C} 1 \\ 2 & \mathrm{H} & \mathrm{H} 2 \\ 3 & \mathrm{C} & \mathrm{C} 2 \\ 4 & \mathrm{H} & \mathrm{H} 4 \\ 5 & \mathrm{P} & \mathrm{P} 1 \\ 6 & \mathrm{H} & \mathrm{H} 1 \\ 7 & \mathrm{C} & \mathrm{C} 3 \\ 8 & \mathrm{C} & \mathrm{C} 4 \\ 9 & \mathrm{H} & \mathrm{H} 3\end{array}$

0.6782409

1.5114805

$-0.6782409$

$-1.5114805$

0.0000000

0.0000000

0.0000000

0.0000000

0.0000000
1. 5916841

2. 2872959

1. 5916841

2. 2872959

0.0000000

$-2.2872959$

$-1.5916841$

$-1.5916841$

$-2.2872959$ 


\section{23-singlet $\mathrm{C}_{4} \mathrm{H}_{6} \mathrm{P}^{+}$}

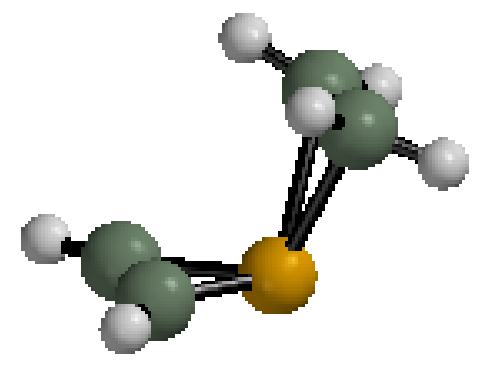

Atom

Cartesian Coordinates (Angstroms)

$\mathrm{X}$

$Y$

$\mathrm{Z}$
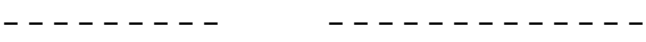

$1 \mathrm{C} \quad \mathrm{C} 1$

1. 7446535

0.6549064

1. 5475279

$-0.2741005$

2. 2871474

$-0.6602974$

0.0160868

$3 \mathrm{C} \quad \mathrm{C} 2$

1. 7434859

$-1.5499195$

$-0.2669851$

2. 2844091

$-0.0065227$

0.0342963

$\begin{array}{lll}5 & \mathrm{P} & \mathrm{P} 1\end{array}$

$6 \mathrm{H}$ H1

$\begin{array}{lll}7 & \mathrm{C} & \mathrm{C} 3\end{array}$

0.3153441

0.9398868

$-1.1431356$

$-0.6436870$

0.0086391

1. 2544661

$-0.9321911$

0.7726507

$-1.7985410$

$-0.0041020$

$-0.2723343$

$-0.6380248$

$-0.9113468$

1. 2720439

$-2.1838995$

0.9175340

$-0.7054612$

$-2.1786965$

$-0.9363057$

$-0.6875271$ 
24. 24-singlet T.S. $\mathrm{C}_{4} \mathrm{H}_{6} \mathrm{P}^{+}$

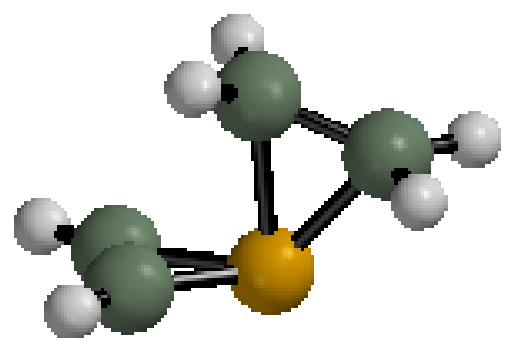

Atom

Cartesian Coordinates (Angstroms)

$\mathrm{X}$

$\mathrm{Y}$

Z

- - - - - - -

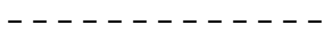

$\begin{array}{llll}1 & \mathrm{C} & \mathrm{C} 1\end{array}$

$2 \mathrm{H} H 2$

3 C $\mathrm{C} 2$

$\begin{array}{lll}4 & \mathrm{H} & \mathrm{H} 4\end{array}$

5 P P1

$6 \mathrm{H} \quad \mathrm{H} 1$

$\begin{array}{lll}7 & \mathrm{C} & \mathrm{C} 3\end{array}$

$8 \mathrm{C} \quad \mathrm{C} 4$

$9 \mathrm{H}$ H3

$10 \mathrm{H}$ H5

$11 \mathrm{H} \quad \mathrm{H} 6$

1. 7774319

0.6660295

1.5441132

.2255403

2.3818461

$-0.6554715$

$-0.0296429$

1.7834846

$-1.5357591$

$-0.2435239$

2.3922148

0.0071745

$-0.0732201$

0.2040222

0.8941748

$-0.7937707$

$-0.8426392$

$-0.9366809$

$-0.0190276$

1.3634655

$-1.7019662$

0.0106790

0.7790605

$-0.8537721$

$-0.9566619$

$-0.4157354$

$-2.0960562$

0.9415384

1.3257109

$-2.1078848$

$-0.8967893$

$-0.8239835$

$-0.8628202$ 
25. 25-singlet $\mathrm{C}_{4} \mathrm{H}_{6} \mathrm{P}^{+}$

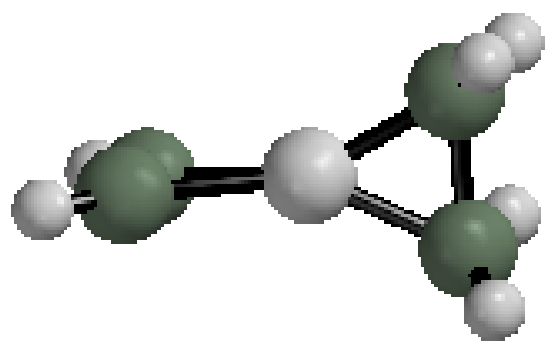

Atom

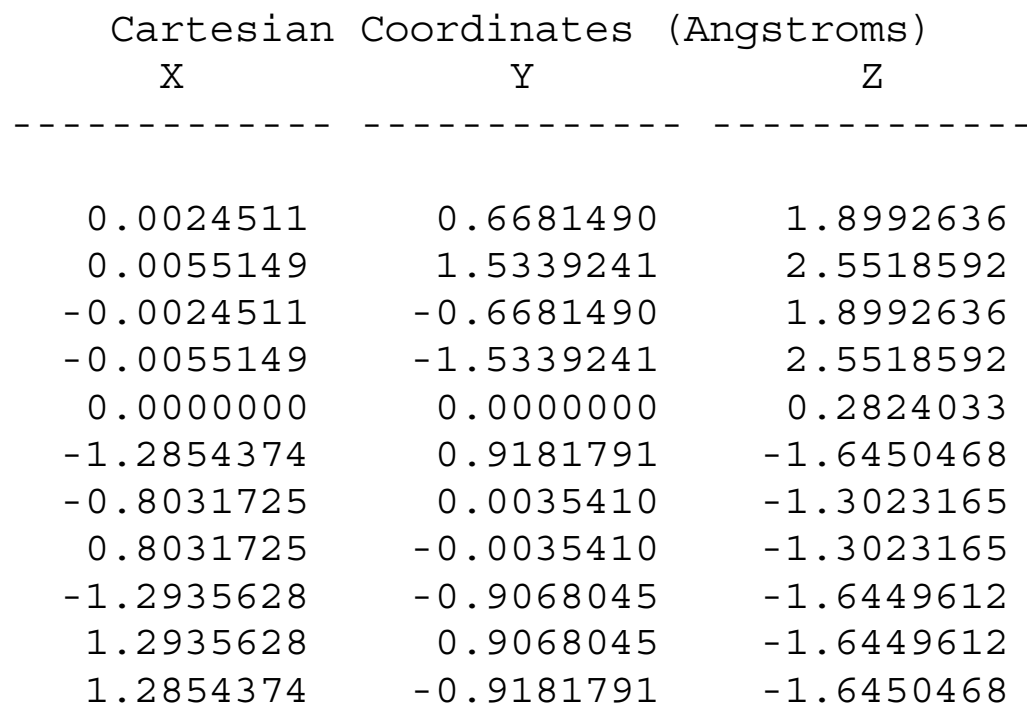

$\begin{array}{lll}1 & \mathrm{C} & \mathrm{C} 1\end{array}$

$2 \mathrm{H} \quad \mathrm{H} 2$

$3 \mathrm{C} \quad \mathrm{C} 2$

$\begin{array}{lll}4 & \mathrm{H} & \mathrm{H} 4\end{array}$

$\begin{array}{lll}5 & \mathrm{P} & \mathrm{P} 1\end{array}$

$6 \mathrm{H} \quad \mathrm{HI}$

$\begin{array}{lll}7 & \mathrm{C} & \mathrm{C} 3\end{array}$

$8 \mathrm{C} \quad \mathrm{C} 4$

$9 \mathrm{H} \quad \mathrm{H3}$

$10 \mathrm{H}$ H5

$11 \mathrm{H} H 6$

1. 2854374

$-0.9181791$

$-1.6450468$

26. 26-singlet $\mathrm{C}_{2} \mathrm{H}_{4}$

$\begin{array}{llll} & & \text { Atom } \\ & 1 & \mathrm{H} & \mathrm{H} 1 \\ 2 & \mathrm{C} & \mathrm{C} 1 \\ 3 & \mathrm{C} & \mathrm{C} 2 \\ 4 & \mathrm{H} & \mathrm{H} 2 \\ 5 & \mathrm{H} & \mathrm{H} 3 \\ 6 & \mathrm{H} & \mathrm{H} 4\end{array}$

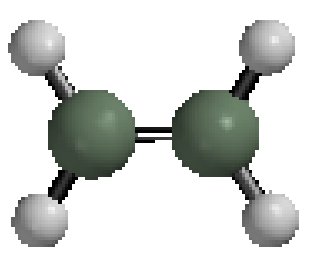

Cartesian Coordinates (Angstroms)

Atom

$$
\mathrm{X}
$$

$\mathrm{Y}$

$\mathrm{Z}$

$\begin{array}{rrr}1.2397166 & -0.9235638 & 0.0000000 \\ 0.6654640 & 0.0000000 & 0.0000000 \\ -0.6654640 & 0.0000000 & 0.0000000 \\ 1.2397166 & 0.9235638 & 0.0000000 \\ -1.2397166 & -0.9235638 & 0.0000000 \\ -1.2397166 & 0.9235638 & 0.0000000\end{array}$


27. 27-Singlet $\mathrm{C}_{4} \mathrm{H}_{6}$

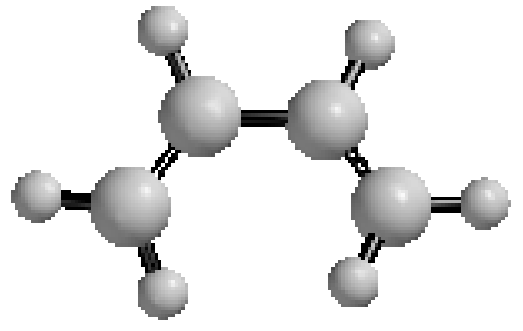

$\begin{array}{crr}\text { Cartesian Coordinates } & \text { (Angstroms) } \\ \mathrm{X} & \mathrm{Y} & \mathrm{Z}\end{array}$

Atom

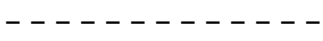

$\begin{array}{rll}1 & \mathrm{C} & \mathrm{C} 2 \\ 2 & \mathrm{C} & \mathrm{C} 3 \\ 3 & \mathrm{C} & \mathrm{C} 1 \\ 4 & \mathrm{C} & \mathrm{C} 4 \\ 5 & \mathrm{H} & \mathrm{H} 3 \\ 6 & \mathrm{H} & \mathrm{H} 4 \\ 7 & \mathrm{H} & \mathrm{H} 1 \\ 8 & \mathrm{H} & \mathrm{H} 6 \\ 9 & \mathrm{H} & \mathrm{H} 2 \\ 10 & \mathrm{H} & \mathrm{H} 5\end{array}$

0.7297509

$-0.0875686$

$-0.6144334$

$-0.7297509$

0.0875686

$-0.6144334$

1. 5428933

0.1030251

0.4324819

$-1.5428933$

$-0.1030251$

0.4324819

1.1697318

$-0.3786247$

$-1.5690276$

$-1.1697318$

0.3786247

1.1694641

0.4461865

$-1.5690276$

$-1.1694641$

$-0.4461865$

1. 3942758

2. 6131427

$-0.0665724$

1. 3942758

$-2.6131427$

0.0665724

0.3567033

0.3567033 
28. 28-Singlet $\mathrm{C}_{6} \mathrm{H}_{8} \mathrm{P}^{+}$

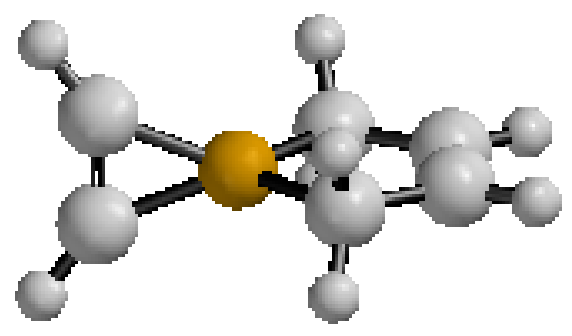

Cartesian Coordinates (Angstroms)

Atom

$\mathrm{X}$

$\mathrm{Y}$

Z

$\begin{array}{rrlrrr}1 & \mathrm{C} & \mathrm{C} 1 & -2.3385269 & -0.6616934 & 0.0000000 \\ 2 & \mathrm{H} & \mathrm{H} 2 & -2.9825349 & -1.5336354 & 0.0000000 \\ 3 & \mathrm{C} & \mathrm{C} 2 & -2.3365371 & 0.6651419 & 0.0000000 \\ 4 & \mathrm{H} & \mathrm{H} 4 & -2.9778154 & 1.5390863 & 0.0000000 \\ 5 & \mathrm{P} & \mathrm{P} 1 & -0.7074188 & -0.0011927 & 0.0000000 \\ 6 & \mathrm{C} & \mathrm{C} 3 & 0.4805900 & -0.0020749 & -1.4058781 \\ 7 & \mathrm{H} & \mathrm{H} 1 & 0.3326650 & 0.8814048 & -2.0390824 \\ 8 & \mathrm{H} & \mathrm{H} 3 & 0.3355129 & -0.8886115 & -2.0354703 \\ 9 & \mathrm{C} & \mathrm{C} 4 & 1.8036829 & 0.0015172 & -0.6689815 \\ 10 & \mathrm{H} & \mathrm{H} 5 & 2.7189658 & 0.0039111 & -1.2531092 \\ 11 & \mathrm{C} & \mathrm{C} 5 & 1.8036829 & 0.0015172 & 0.6689815 \\ 12 & \mathrm{H} & \mathrm{H} 6 & 2.7189658 & 0.0039111 & 1.2531092 \\ 13 & \mathrm{C} & \mathrm{C} 6 & 0.4805900 & -0.0020749 & 1.4058781 \\ 14 & \mathrm{H} & \mathrm{H} 7 & 0.3355129 & -0.8886115 & 2.0354703 \\ 15 & \mathrm{H} & \mathrm{H} 8 & 0.3326650 & 0.8814048 & 2.0390824\end{array}$


29. 29-Singlet $\mathrm{C}_{6} \mathrm{H}_{8} \mathrm{P}^{+}$

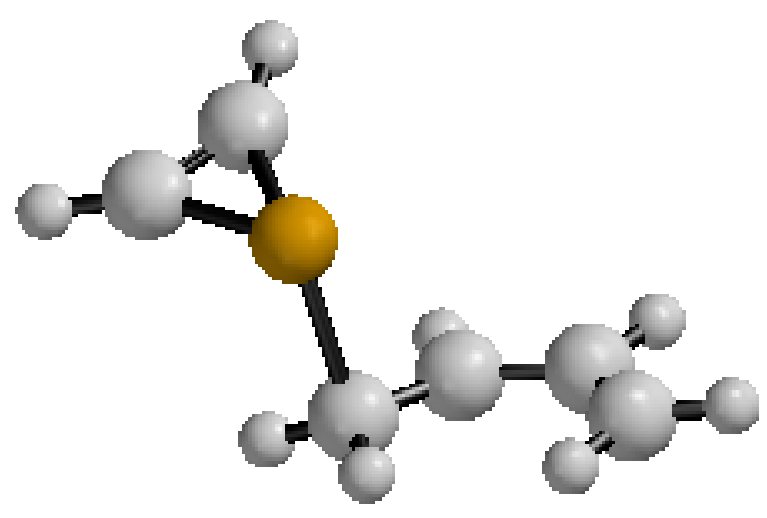

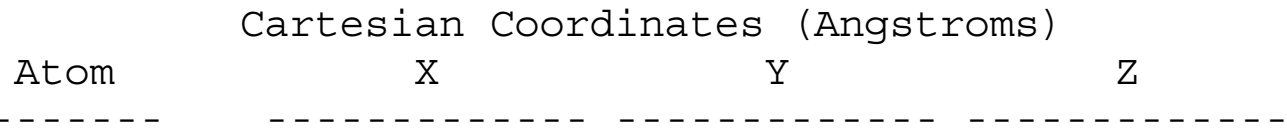

$\begin{array}{rrrrrr}1 & \mathrm{C} & \mathrm{C} 1 & 2.4437755 & 0.9348581 & -0.5706448 \\ 2 & \mathrm{H} & \mathrm{H} 2 & 2.6168051 & 1.9991935 & -0.4671493 \\ 3 & \mathrm{C} & \mathrm{C} 2 & 2.9752870 & -0.2597579 & -0.4400436 \\ 4 & \mathrm{H} & \mathrm{H} 4 & 3.8595154 & -0.8097244 & -0.1413068 \\ 5 & \mathrm{P} & \mathrm{P} 1 & 1.3050338 & -0.3540450 & -1.0937139 \\ 6 & \mathrm{C} & \mathrm{C} 3 & 0.0524340 & -0.9391307 & 0.6142204 \\ 7 & \mathrm{H} & \mathrm{H} 1 & 0.8110851 & -1.2075752 & 1.3469683 \\ 8 & \mathrm{H} & \mathrm{H} 3 & -0.4452099 & -1.7884447 & 0.1483533 \\ 9 & \mathrm{C} & \mathrm{C} 4 & -0.6662418 & 0.2375223 & 0.8416676 \\ 10 & \mathrm{H} & \mathrm{H} 5 & -0.2069245 & 0.9688855 & 1.5064813 \\ 11 & \mathrm{C} & \mathrm{C} 5 & -1.8972794 & 0.6450396 & 0.2254795 \\ 12 & \mathrm{H} & \mathrm{H} 6 & -2.2332966 & 1.6562590 & 0.4385870 \\ 13 & \mathrm{C} & \mathrm{C} 6 & -2.6513322 & -0.1421703 & -0.5792688 \\ 14 & \mathrm{H} & \mathrm{H} 7 & -2.3957169 & -1.1739823 & -0.8051342 \\ 15 & \mathrm{H} & \mathrm{H} 8 & -3.5679347 & 0.2330725 & -1.0244961\end{array}$


30. 30-Singlet T. S. $\quad \mathrm{C}_{6} \mathrm{H}_{8} \mathrm{P}^{+}$

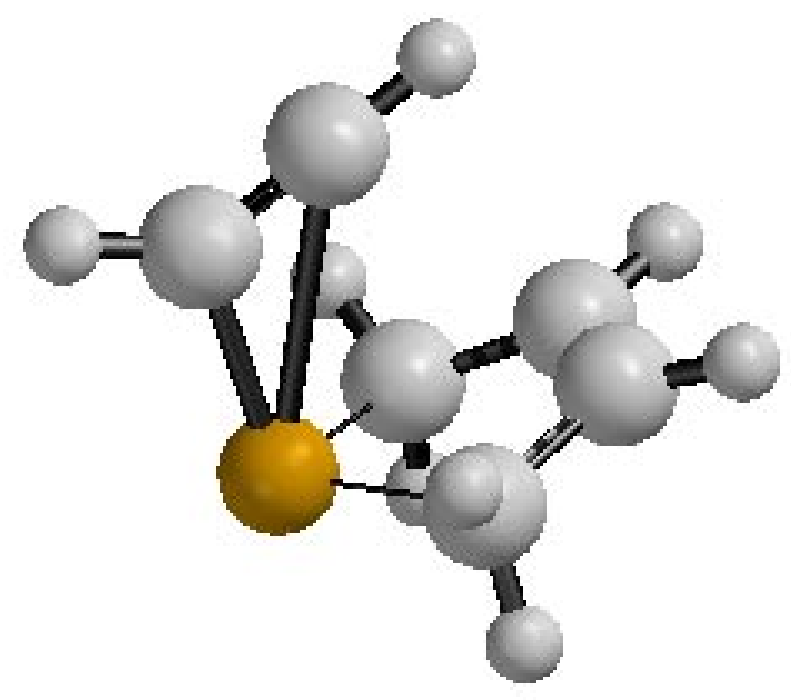

Atom

Cartesian Coordinates (Angstroms)

$$
\mathrm{X}
$$

$\mathrm{Y}$

$\mathrm{Z}$

$\begin{array}{rrlrrr}1 & \mathrm{C} & \mathrm{C} 3 & 0.1099655 & 0.6965517 & 1.5328672 \\ 2 & \mathrm{C} & \mathrm{C} 4 & 0.1099655 & -0.6965517 & 1.5328672 \\ 3 & \mathrm{C} & \mathrm{C} 5 & -0.4023260 & 1.3510605 & 0.4049889 \\ 4 & \mathrm{C} & \mathrm{C} 6 & -0.4023260 & -1.3510605 & 0.4049889 \\ 5 & \mathrm{H} & \mathrm{H} 3 & 0.5072517 & 1.2599781 & 2.3716106 \\ 6 & \mathrm{H} & \mathrm{H} 1 & 0.5072517 & -1.2599781 & 2.3716106 \\ 7 & \mathrm{H} & \mathrm{H} 5 & -1.2824507 & 1.9352468 & 0.6922672 \\ 8 & \mathrm{H} & \mathrm{H} 6 & -1.2824507 & -1.9352468 & 0.6922672 \\ 9 & \mathrm{H} & \mathrm{H} 8 & 0.3227189 & 2.0623823 & -0.0022708 \\ 10 & \mathrm{H} & \mathrm{H} 9 & 0.3227189 & -2.0623823 & -0.0022708 \\ 11 & \mathrm{P} & \mathrm{P} 1 & -0.8721705 & 0.0000000 & -0.7557598 \\ 12 & \mathrm{C} & \mathrm{C} 1 & -0.2074014 & 0.0000000 & -2.2271102 \\ 13 & \mathrm{H} & \mathrm{H} 2 & -0.5318466 & 0.0000000 & -3.2538687 \\ 14 & \mathrm{C} & \mathrm{C} 2 & 1.0192372 & 0.0000000 & -1.7982503 \\ 15 & \mathrm{H} & \mathrm{H} 7 & 2.0818625 & 0.0000000 & -1.9639370\end{array}$




\section{31-Singlet $\mathrm{C}_{6} \mathrm{H}_{8} \mathrm{P}^{+}$}

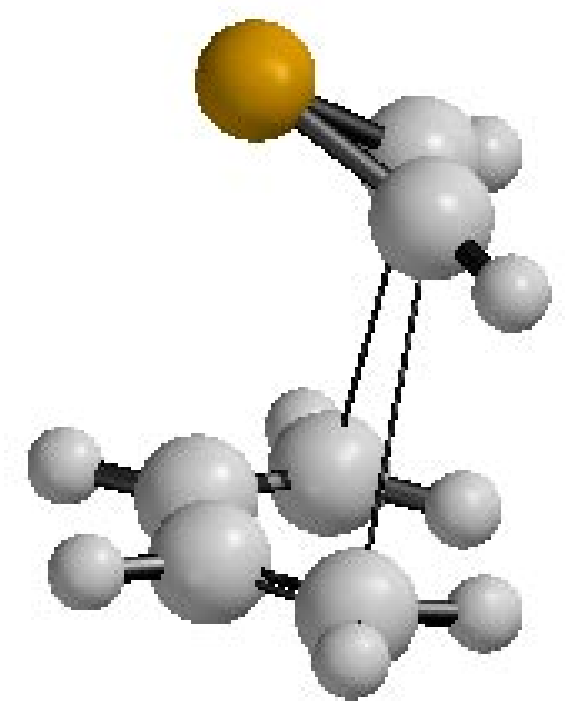

Atom

Cartesian Coordinates (Angstroms) - Atom $\mathrm{X}$ $\mathrm{Y}$

Z

$\begin{array}{rllrrr}1 & \mathrm{H} & \mathrm{H} 1 & 0.6183352 & -1.4925667 & 1.1677888 \\ 2 & \mathrm{C} & \mathrm{C} 1 & 0.8669597 & -0.5022051 & 1.5437974 \\ 3 & \mathrm{C} & \mathrm{C} 3 & 1.1936781 & 0.5233936 & -0.7323602 \\ 4 & \mathrm{C} & \mathrm{C} 5 & -2.0026232 & 0.1027192 & -0.6813649 \\ 5 & \mathrm{C} & \mathrm{C} 4 & 0.8669597 & -0.5022051 & -1.5437974 \\ 6 & \mathrm{C} & \mathrm{C} 6 & -2.0026232 & 0.1027192 & 0.6813649 \\ 7 & \mathrm{C} & \mathrm{C} 2 & 1.1936781 & 0.5233936 & 0.7323602 \\ 8 & \mathrm{H} & \mathrm{H} 7 & 0.6183352 & -1.4925667 & -1.1677888 \\ 9 & \mathrm{H} & \mathrm{H} 11 & -1.9525120 & -0.5267432 & 1.5596717 \\ 10 & \mathrm{H} & \mathrm{H} 3 & 1.4959600 & 1.4614265 & 1.1965744 \\ 11 & \mathrm{H} & \mathrm{H} 2 & 0.9246525 & -0.3989783 & 2.6233687 \\ 12 & \mathrm{H} & \mathrm{H} 5 & 1.4959600 & 1.4614265 & -1.1965744 \\ 13 & \mathrm{H} & \mathrm{H} 9 & -1.9525120 & -0.5267432 & -1.5596717 \\ 14 & \mathrm{H} & \mathrm{H} 8 & 0.9246525 & -0.3989783 & -2.6233687 \\ 15 & \mathrm{P} & \mathrm{P} 1 & -2.2889005 & 1.6659082 & 0.0000000\end{array}$




\section{32-Singlet T. S. $\quad \mathrm{C}_{6} \mathrm{H}_{8} \mathrm{P}^{+}$}

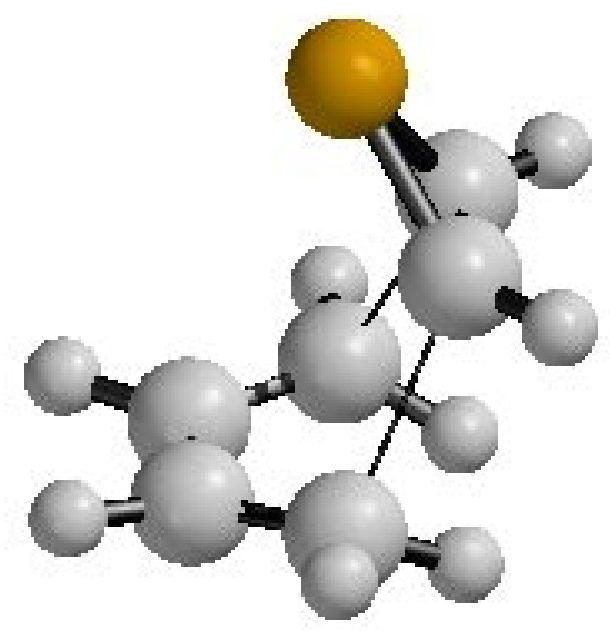

Cartesian Coordinates (Angstroms)

Atom $\mathrm{X}$ $\mathrm{Y}$

$\begin{array}{rll}1 & \mathrm{H} & \mathrm{H} 1 \\ 2 & \mathrm{C} & \mathrm{C} 1 \\ 3 & \mathrm{C} & \mathrm{C} 3 \\ 4 & \mathrm{C} & \mathrm{C} 5 \\ 5 & \mathrm{C} & \mathrm{C} 4 \\ 6 & \mathrm{C} & \mathrm{C} 6 \\ 7 & \mathrm{C} & \mathrm{C} 2 \\ 8 & \mathrm{H} & \mathrm{H} 7 \\ 9 & \mathrm{H} & \mathrm{H} 11 \\ 10 & \mathrm{H} & \mathrm{H} 3 \\ 11 & \mathrm{H} & \mathrm{H} 2 \\ 12 & \mathrm{H} & \mathrm{H} 5 \\ 13 & \mathrm{H} & \mathrm{H} 9 \\ 14 & \mathrm{H} & \mathrm{H} 8 \\ 15 & \mathrm{P} & \mathrm{P} 1\end{array}$

$$
\begin{array}{r}
-1.4130533 \\
-0.4768722 \\
0.1825994 \\
0.3523207 \\
-0.4768722 \\
0.3523207 \\
0.1825994 \\
-1.4130533 \\
-0.1674107 \\
0.8925660 \\
-0.3355939 \\
0.8925660 \\
-0.1674107 \\
-0.3355939 \\
1.9308880
\end{array}
$$

0.0043511

$-0.4176541$

$-1.3820678$

1.4288936

$-0.4176541$

1. 4288936

$-1.3820678$

0.0043511

1.9704457

$-2.0347389$

$-0.3839253$

$-2.0347389$

1.9704457

$-0.3839253$

1.6293915

1.0981094

1.4526366

$-0.7055060$

$-0.7169588$

$-1.4526366$

0.7169588

0.7055060

$-1.0981094$

1.4995167

1. 2096239

2.5293555

$-1.2096239$

$-1.4995167$

$-2.5293555$

0.0000000 
33. 33-Singlet $\mathrm{C}_{6} \mathrm{H}_{8} \mathrm{P}^{+}$

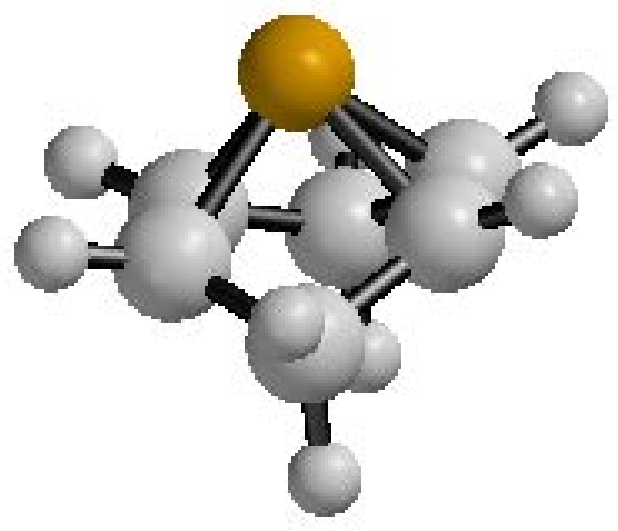

Atom

Cartesian Coordinates (Angstroms)

Atom

$\begin{array}{rll}1 & \mathrm{H} & \mathrm{H} 1 \\ 2 & \mathrm{C} & \mathrm{C} 1 \\ 3 & \mathrm{C} & \mathrm{C} 3 \\ 4 & \mathrm{C} & \mathrm{C} 5 \\ 5 & \mathrm{C} & \mathrm{C} 4 \\ 6 & \mathrm{C} & \mathrm{C} 6 \\ 7 & \mathrm{C} & \mathrm{C} 2 \\ 8 & \mathrm{H} & \mathrm{H} 7 \\ 9 & \mathrm{H} & \mathrm{H} 11 \\ 10 & \mathrm{H} & \mathrm{H} 3 \\ 11 & \mathrm{H} & \mathrm{H} 2 \\ 12 & \mathrm{H} & \mathrm{H} 5 \\ 13 & \mathrm{H} & \mathrm{H} 9 \\ 14 & \mathrm{H} & \mathrm{H} 8 \\ 15 & \mathrm{P} & \mathrm{P} 1\end{array}$

$-1.6128341$

$-0.0000014$

1.2615961

$-0.5268021$

$-0.0000860$

1.4142695

0.2054188

$-1.1340756$

$-0.7131800$

0.2048069

1.1340441

$-0.7130197$

$-0.5268021$

$-0.0000860$

$-1.4142695$

0.2048069

1.1340441

0.7130197

0.2054188

$-1.1340756$

0.7131800

$-1.6128341$

$-0.0000014$

$-1.2615961$

0.5786616

1.9968770

1. 2590906

0.5782884

$-1.9972724$

1. 2592554

$-0.3198061$

0.0003715

2. 4849661

0.5782884

$-1.9972724$

$-1.2592554$

0.5786616

1. 9968770

$-1.2590906$

$-0.3198061$

0.0003715

1. 7845332

0.0002857

$-2.4849661$

0.0000000 
34. 34-Singlet $\quad \mathrm{C}_{4} \mathrm{H}_{6}$

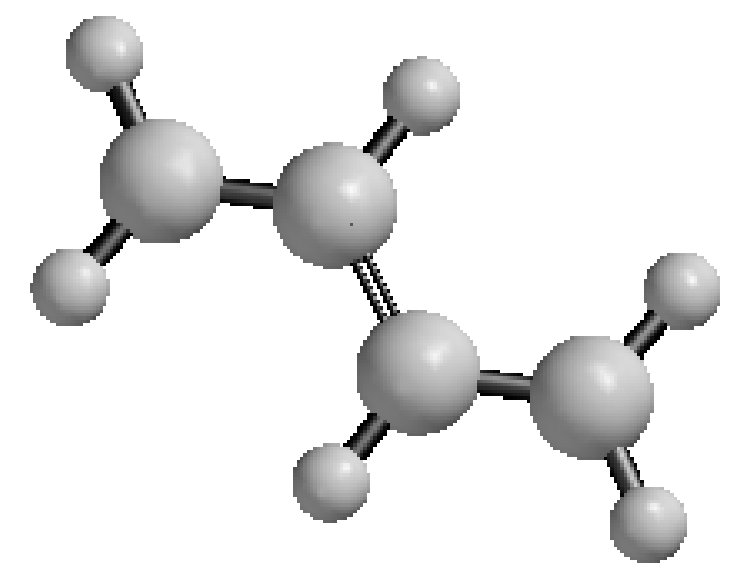

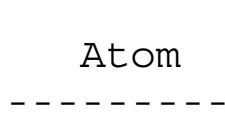

$\begin{array}{lll}1 & \mathrm{H} & \mathrm{H} 1\end{array}$

$\begin{array}{lll}2 & \mathrm{C} & \mathrm{C} 1\end{array}$

$3 \mathrm{H} \quad \mathrm{H} 2$

$4 \mathrm{C} \quad \mathrm{C} 2$

$5 \mathrm{H} \quad \mathrm{H3}$

$6 \mathrm{C} \quad \mathrm{C} 3$

$\begin{array}{lll}7 & \mathrm{H} & \mathrm{H} 4\end{array}$

$8 \mathrm{C} \quad \mathrm{C} 4$

$9 \mathrm{H}$ H5

$10 \mathrm{H} \mathrm{H6}$

. 


\section{35-Singlet $\quad \mathrm{C}_{6} \mathrm{H}_{8} \mathrm{P}^{+}$}

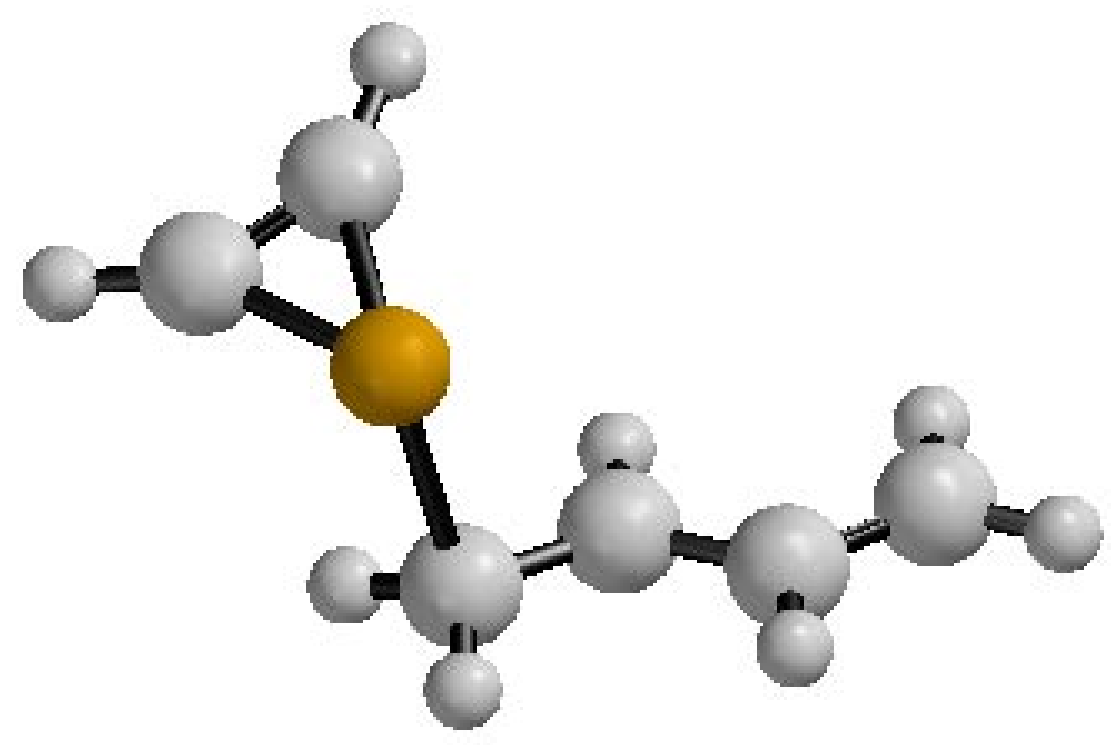

\begin{tabular}{rllrrr}
\multicolumn{1}{c}{ Atom } & \multicolumn{2}{c}{ Cartesian Coordinates } & \multicolumn{2}{c}{ (Angstroms) } \\
& & X & \multicolumn{1}{c}{ Y } \\
1 & C & C3 & -1.9296364 & 0.3049471 & 0.2595702 \\
2 & C & C1 & -0.7289854 & 0.3247271 & -0.5121548 \\
3 & H & H3 & -0.6097666 & -0.4618752 & -1.2580741 \\
4 & C & C2 & 0.3160079 & 1.2332692 & -0.3377730 \\
5 & H & H2 & 1.0352259 & 1.3428406 & -1.1468772 \\
6 & H & H8 & 0.1175176 & 2.1424965 & 0.2296558 \\
7 & P & P1 & 1.6051589 & 0.2104563 & 1.1574568 \\
8 & C & C5 & 2.3375570 & -1.2322764 & 0.3740302 \\
9 & H & H1 & 2.2511735 & -2.2928935 & 0.1718814 \\
10 & C & C6 & 3.1025451 & -0.1717651 & 0.2429696 \\
11 & H & H11 & 4.0389326 & 0.2054743 & -0.1499074 \\
12 & H & H12 & -2.0800075 & 1.0816684 & 1.0064569 \\
13 & C & C4 & -2.8828317 & -0.6347835 & 0.0496407 \\
14 & H & H4 & -2.7621891 & -1.4129117 & -0.7006155 \\
15 & H & H5 & -3.8107018 & -0.6393740 & 0.6137405
\end{tabular}


36. 36-Singlet T. S. $\quad \mathrm{C}_{6} \mathrm{H}_{8} \mathrm{P}^{+}$

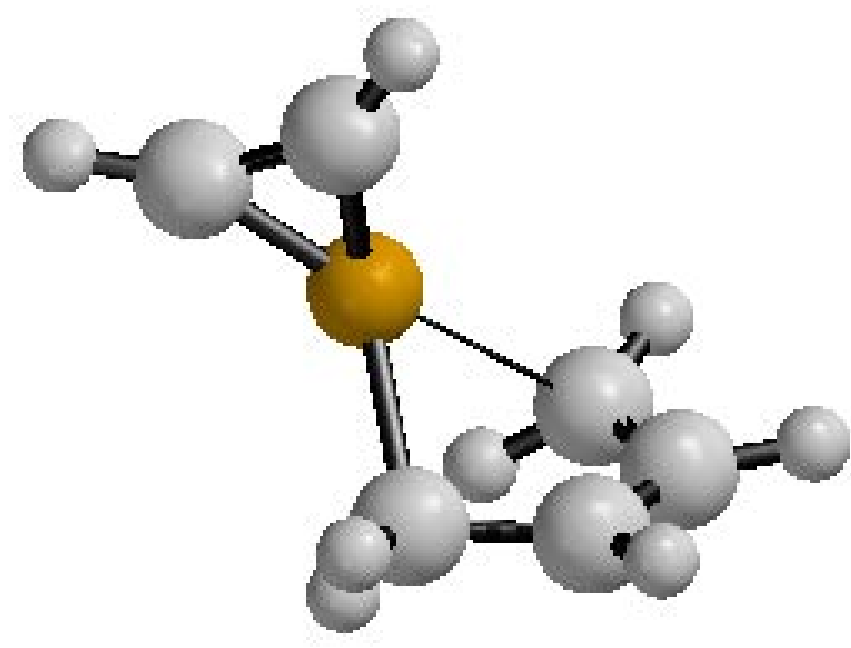

Atom

Cartesian Coordinates (Angstroms) Atom..--

$\begin{array}{rll}1 & \mathrm{C} & \mathrm{C} 3 \\ 2 & \mathrm{C} & \mathrm{C} 1 \\ 3 & \mathrm{H} & \mathrm{H} 3 \\ 4 & \mathrm{C} & \mathrm{C} 2 \\ 5 & \mathrm{H} & \mathrm{H} 2 \\ 6 & \mathrm{H} & \mathrm{H} 8 \\ 7 & \mathrm{P} & \mathrm{P} 1 \\ 8 & \mathrm{C} & \mathrm{C} 5 \\ 9 & \mathrm{H} & \mathrm{H} 1 \\ 10 & \mathrm{C} & \mathrm{C} 6 \\ 11 & \mathrm{H} & \mathrm{H} 11 \\ 12 & \mathrm{H} & \mathrm{H} 12 \\ 13 & \mathrm{C} & \mathrm{C} 4 \\ 14 & \mathrm{H} & \mathrm{H} 4 \\ 15 & \mathrm{H} & \mathrm{H} 5\end{array}$

1.9247180
1.2151883
1.3430102
0.0884463
0.3203813
-0.5330822
-0.8853130
-2.6741320
-3.5804990
-2.2098367
-2.4972195
2.5562921
1.6309103
1.9243095
1.3768263

$-0.0860223$

$-0.4342460$

1.0947977

$-0.2532636$

1.8932157

$-0.9805571$

1.1568124

0.6566562

0.9433128

1.7056967

2.0491291

0.5733857

$-0.5445022$

0.2152039

$-0.4995970$

0.2678923

$-0.5351536$

0.8602120

$-0.3928806$

$-0.9647736$

$-0.2879116$

$-2.0043469$

$-0.1885661$

$-1.2249711$

$-1.3132276$

$-2.2112875$

0.3228797

$-0.0274937$

$-1.1663760$

1.3759820 
Table S1. Total and relative energies of $\mathrm{C}_{2} \mathrm{H}_{2} \mathrm{P}^{+}$isomers at the G3//B3LYP/6-31G* level.

\begin{tabular}{|c|c|c|}
\hline species & Energy (a.u.) at G3//B3LYP/6-31G* & Relative energy (kcal/mol) \\
\hline $\mathbf{1}$ & -418.19544 & 0.0 \\
\hline $\mathbf{2}$ & -418.16674 & 18.0 \\
\hline $\mathbf{3}$ & -418.13731 & 36.5 \\
\hline $\mathbf{4}$ & -418.120176 & 47.2 \\
\hline $\mathbf{5}$ & -418.10574 & 56.3 \\
\hline $\mathbf{6}$ & -418.06380 & 84.6 \\
\hline $\mathbf{7}$ & -418.06090 & 100.0 \\
\hline $\mathbf{8}$ & -418.03600 & 108.5 \\
\hline $\mathbf{9}$ & -418.02258 & 110.2 \\
\hline $\mathbf{1 0}$ & -418.01975 & \\
\hline
\end{tabular}


Table S2. Total energies of species involved in the potential energy surfaces of the reaction of $\mathrm{PBr}^{+}$with $\mathrm{C}_{2} \mathrm{H}_{2}$.

\begin{tabular}{|c|c|c|}
\hline species & Energy at G3//B3LYP/6-31G* & Energy at B3LYP/6-311+G**//B3LYP/6-31G* \\
\hline $\mathbf{1}$ & -418.19544 & -418.44066 \\
\hline $\mathbf{2}$ & -418.16674 & -418.41753 \\
\hline $\mathbf{1 1}$ & -77.27584 & -77.35657 \\
\hline $\mathbf{1 2}$ & -2914.40126 & -2915.15160 \\
\hline $\mathbf{1 3}$ & -2573.51316 & -2574.10358 \\
\hline $\mathbf{1 4}$ & -2991.71756 & -2992.55288 \\
\hline $\mathbf{1 5}$ & -2991.71849 & -2992.55208 \\
\hline $\mathbf{1 6}$ & -2991.74491 & -2992.57433 \\
\hline $\mathbf{1 7}$ & -2991.71034 & -2992.54064 \\
\hline $\mathbf{1 8}$ & -2991.74414 & -2992.58288 \\
\hline
\end{tabular}


Table S3. Total energies of species involved in the potential energy surfaces of the reaction of $\mathrm{C}_{2} \mathrm{H}_{2} \mathrm{P}^{+}$with $\mathrm{C}_{4} \mathrm{H}_{6}$.

\begin{tabular}{|c|c|}
\hline species & Energy at G3//B3LYP/6-31G* \\
\hline $\mathbf{1}$ & -418.19544 \\
\hline $\mathbf{2 7}$ & -155.84046 \\
\hline $\mathbf{2 8}$ & -574.12577 \\
\hline $\mathbf{2 9}$ & -574.07165 \\
\hline $\mathbf{3 0}$ & -574.06652 \\
\hline $\mathbf{3 1}$ & -574.05136 \\
\hline $\mathbf{3 2}$ & -574.03294 \\
\hline $\mathbf{3 3}$ & -574.13037 \\
\hline $\mathbf{3 4}$ & -155.84515 \\
\hline $\mathbf{3 5}$ & -574.07575 \\
\hline $\mathbf{3 6}$ & -574.06670 \\
\hline
\end{tabular}

